\title{
Selective Radiotherapy after Distant Metastasis of Nasopharyngeal Carcinoma Treated with Dose-Dense Cisplatin plus Fluorouracil
}

\author{
Yong Liang ${ }^{1 *}$, Jun-Guo Bu${ }^{2}$, Jin-ling Cheng ${ }^{1}$, Wei-Wei Gao ${ }^{1}$, Yao-Can $\mathrm{Xu}^{1}$, Jian \\ Feng $^{1}$, Bo-Yu Chen ${ }^{1}$, Wei-Chao Liang ${ }^{1}$, Ke-Quan Chen ${ }^{1}$
}

\begin{abstract}
Purpose: To investigate the efficacy and safety of selective radiotherapy after distant metastasis of nasopharyngeal carcinoma (NPC) treated with dose-dense cisplatin plus fluorouracil. Materials and Methods: Eligible patients were randomly assigned to a study group treated with dose-dense cisplatin plus fluorouracil following selective radiotherapy and a control group receiving traditional cisplatin plus fluorouracil following selective radiotherapy according to a 1:1 distribution using a digital random table method. The primary endpoint was overall survival (OS). Secondary endpoints were progression-free survival (PFS), objective response rate, relapse or progression rate in the radiation field and treatment toxicity. Results: Of 52 patients in the study group, 20 cases underwent radiotherapy., while in the control group of 51 patients, 16 underwent radiotherapy. The median PFS, median OS, survival rates in 1, 2 and 3 years in study and control group were 20.9 vs 12.7months,

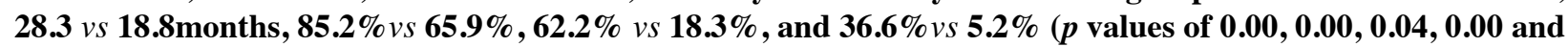
0.00 , respectively). Subgroup analysis showed that the median $O S$ and survival rates of $1,2,3$ years for patients undergoing radiotherapy in the study group better than that in control group (43.2vs24.1 months, 94.1\% vs $86.7 \%, 82.4 \%$ vs $43.3 \%, 64.7 \%$ vs $17.3 \%,(p=0.00,0.57,0.04$ and 0.01 , respectively). The complete response rate, objective response rate after chemotherapy and three months after radiotherapy, relapse or progression rate in radiation field in study group and in control group were $19.2 \%$ vs $\mathbf{3 . 9 \%}, \mathbf{8 6 . 5 \%}$ vs $\mathbf{5 6 . 9 \% , 8 5 \%}$ vs $\mathbf{5 0 \%}$, $95 \%$ vs $81.3 \%$ and $41.3 \%$ vs $66.7 \%(p=0.03,0.00,0.03,0.30,0.01$ respectively). The grade $3-4$ acute adverse reactions in

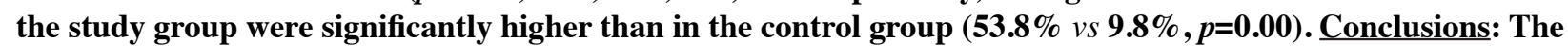
survival of patients benefits from selective radiotherapy after distant metastasis of NPC treated with dose-dense cisplatin plus fluorouracil.
\end{abstract}

Keywords: Nasopharyngeal carcinoma - distant metastases - dose dense chemotherapy - radiotherapy

Asian Pac J Cancer Prev, 16 (14), 6011-6017

\section{Introduction}

The incidence of NPC is high in China and Southeast Asian countries, especially Pearl River Basin of Guangxi and Guangdong. The annual incidence rate is as high as 20/100, 000 (Jemal et al., 2011). The local area is one of the high incidence areas in China. The progress of radiotherapy technology increased the local control rate by 75-95\% (Ng et al., 2010; Xiao et al., 2011; Abbasi et al., 2013). There were about $40 \%$ of patients with unsuccessful local therapy combined with distant metastasis; The distant metastasis still appeared on $18.5 \%$ of patients with local control (Kong et al., 2014); In addition, about $95 \%$ of NPC was undifferentiated non-keratinizing carcinoma. Its biological characteristics are highly invasive and distant metastasis easily occurs (Lee et al., 2012). The distant metastasis occurred on about $20 \%$ of newly diagnosed patients (Xiao et al., 2011). Chemotherapy is the main method for treating distant metastasis of NPC and various kinds of cytotoxic drugs have anticancer activity to NPC. Although there are many drugs in recent years, cisplatin is the most effective drug treatment for NPC now (Kua, et al., 2013). The response rate and progression-free survival time of cisplatin in combination with docetaxel or gemcitabine scheme was not superior to those of cisplatin in combination with fluorouracil (Kertmen et al., 2015). Therefore, cisplatin in combination with fluorouracil remains the standard chemotherapy scheme of NPC. Its objective response rate was about 60\% (Wang et al., 1991). But the progression-free survival and overall survival were still low. The progression-free survival was from 5.6 to 10.6 months and the overall survival was from 7.6 to 19.6 months.

In 1979 , the probability of drug resistance was 
calculated by Goldie and Coldman from cell proliferation dynamics perspective. The tumor cells had a certain probability of mutation in cell proliferation process, leading to the production of drug-resistant cells. The more the doubling times were, the more the drug-resistant cells produced. Shortening the interval of chemotherapy could reduce the production of drug-resistant cells. This viewpoint was being confirmed by more and more clinical practice. For example, doxorubicin, cyclophosphamide and paclitaxel sequential treatment scheme was modified into dose dense in the treatment of high-risk postoperative breast cancer patients. The patient's disease-free survival rate and total survival rate were improved (Hudis et al., 2015). The dose-dense chemotherapy also improved overall survival rate in lymphoma (Aoki et al., 2013), ovarian cancer (Kumar et al., 2015).

Dose-dense chemotherapy is one of the major progresses in chemotherapy achieved in recent years (Hudis et al., 2015). The patients with NPC of the distant metastasis whose lesion was not disseminated and the objective response was better after dose-dense cisplatin combined with fluorouracil chemotherapy, was selected to treat by radiotherapy. The overall survival was significantly increased. The similar report was not retrieved. The results were summarized as follows.

\section{Materials and Methods}

\section{Inclusion criteria of patients}

All enrolled patients were admitted in the hospital and met the following conditions at the same time from April 1 st 2010 to July 30th 2014. (1)The pathological diagnosis was NPC. CT/ECT/MRI/ B ultrasound showed metastatic carcinoma; (2)There was at least one measurable lesion by imaging examination; (3)The age was 18-70 years old; (4)The patients or clients signed the informed consent which was agreed by hospital medical ethics committee and were willing to accept follow-up; (5) The expectancy of life was greater than 3 months and ECOG performance status score was $0-2$; (6)No chronic pulmonary or heart disease. The heart and lung function was normal; (7) Blood test and chemistry profile: $\mathrm{Hb}>100 \mathrm{~g} / \mathrm{L}$, neutrophil $>1.5 * 10^{9} / \mathrm{L}$, platelet $>100 * 10^{9} / 1$, serum creatinine $<$ the upper limit of normal value, serum total bilirubin $<$ the upper limit of normal value, alanine aminotransferase $<2$ times of the upper limit of normal value; (8) The patients who received radiotherapy should meet additionally 2 conditions: Firstly, the lesion was not disseminated and disappeared or reduced after 4-6 cycles of chemotherapy. Secondly, accepting and completing radiotherapy.

\section{Exclusion criteria of patients}

One of the following cases should be excluded: (1) NPC in combination with brain metastasis; (2) Receiving chemotherapy within 6 months; (3) Being allergic to cisplatin and fluorouracil; (4) With a hepatitis or nephritis history; (5) The pregnant or lactating patients.

\section{Randomization}

Of 123 patients involved in the qualification evaluation, 20 patients did not meet the qualification, and 103 patients were eligible. The patients were randomly divided into two groups, and 52 patients in study group receiving radiotherapy after dose-dense chemotherapy, while the other 51 patients in control group receiving radiotherapy after traditional chemotherapy according to $1: 1$ using digital random table method. The baseline features of patients in two groups meeting statistical analysis requirements are listed in Table 1.

\section{Chemotherapy schedule}

Patients were treated with cisplatin in combination with fluorouracil chemotherapy. Routine hydration and antiemetic were performed for 6 cycles or until progression of disease, intolerable side effects or death. Control group: Cisplatin $80 \mathrm{mg} / \mathrm{m}^{2}$ was intravenously injected on day1 of a 4-week cycle; fluorouracil 4000 $\mathrm{mg} / \mathrm{m}^{2}$ continuous infusion for 96 hours of a 4 -week

Table 1. Patients Baseline Characteristics n(\%)

\begin{tabular}{|c|c|c|c|}
\hline Characteristics of patients & $\begin{array}{c}\text { Study group } \\
n=52\end{array}$ & $\begin{array}{c}\text { Control group } \\
n=51\end{array}$ & $p$ value \\
\hline Average age(range) & $46.8(27 \sim 70)$ & $44.2(34 \sim 69)$ & 0.07 \\
\hline Sex(male/ female) & $41 / 11$ & $39 / 12$ & 0.82 \\
\hline Pathological types WHOII/III & $2 / 50$ & $4 / 47$ & 0.44 \\
\hline Nasopharyngeal lesions with/without & $30 / 22$ & $28 / 23$ & 0.84 \\
\hline Single/multiple organ metastasis & $11 / 41$ & $15 / 36$ & 0.37 \\
\hline Metastatic site & & & 0.78 \\
\hline Bone metastasis & $30(57.7)$ & $33(64.7)$ & \\
\hline Hepatic metastasis & $19(36.5)$ & $15(29.4)$ & \\
\hline Pulmonary metastasis & $10(19.2)$ & $13(25.5)$ & \\
\hline Abdominal lymph node metastasis & $6(11.5)$ & $5(9.8)$ & \\
\hline Primary treatment & $28(53.8)$ & $25(49.0)$ & 0.89 \\
\hline Radiotherapy history & $24(46.2)$ & $26(51.0)$ & \\
\hline Chemotherapy history & $18(34.6)$ & $18(35.3)$ & \\
\hline Chemotherapy drugs & & & 0.79 \\
\hline Platinum & $18(34.6)$ & $18(35.3)$ & \\
\hline Paclitaxel & $12(23)$ & $10(19.6)$ & \\
\hline Gemcitabine & $6(11.5)$ & $8(15.7)$ & \\
\hline ECOG performance status score $(0 / 1 / 2)$ & $8 / 28 / 16$ & $10 / 30 / 11$ & 0.55 \\
\hline
\end{tabular}

Abbreviations: ECOG, Eastern Cooperative Oncology Group; WHO, World Health Organization 
cycle. Study group: Cisplatin $80 \mathrm{mg} / \mathrm{m}^{2}$ was intravenously injected on day 1 of a 2 -week cycle; fluorouracil 4000 $\mathrm{mg} / \mathrm{m}^{2}$ continuous infusion for 96 hours of a 2 -week cycle; 300ug recombinant human granulocyte colony stimulating factor was subcutaneously injected, days6-10. Those patients receiving radiotherapy were intravenously injected with $30 \mathrm{mg} / \mathrm{m}^{2}$ cisplatin weekly during the period of radiotherapy

\section{Radiotherapy scheme}

The enrolled patients underwent three dimensional conformal radiotherapy at 3 4 weeks after chemotherapy by using Elekta 6MV X-ray. The patients were immobilized in the supine position with a thermoplastic mask and vacuum bag. CT scan thickness: head and neck $3 \mathrm{~mm}$, chest, abdomen and limbs $5 \mathrm{~mm}$. The workstation was Elekta Precise Plan R2.15 three-dimensional treatment planning system. The delineation of target and organ at risk referred to the definition ICRU No. 83 report. Gross tumor volume (GTV) was residual tumor showed by CT and MRI after chemotherapy; clinic tumor volume (CTV) was gross tumor extended to $3 \sim 10 \mathrm{~mm}$ showed by MRI/ CT before chemotherapy. Special circumstances that if vertebral metastasis CTV included the entire vertebral body, neck lymph nodes metastasis included the drainage area of the lymph node, and lesion had bone barrier, it was generally extended to $3 \mathrm{~mm}$; planning target volume (PTV) had a great difference between the different parts according to respiratory motion size and posture repeatability. Generally, the head and neck extended to $3 \sim 5 \mathrm{~mm}$, chest, abdomen and limbs extended to $8 \sim 15 \mathrm{~mm}$. Nasopharyngeal or neck radiotherapy dose: if the local and distant lesion disappeared, primary tumor bed for a total
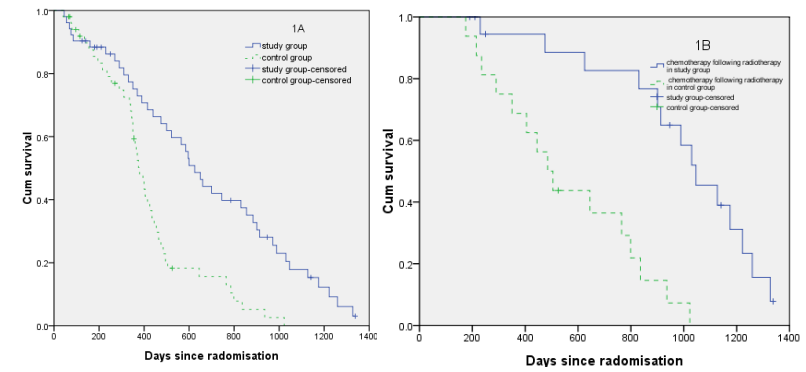

Figure 1. Kaplan-Meier Curve of Progression-Free Survival (PFS) 1A Comparisons between Study and Control Group, 1B Comparisons between Chemotherapy Following Radiotherapy in Study and Control Group dose 60 Gy. Nevertheless, if the local residual and distal lesion disappeared, local for total dose 70 Gy. Supposing the local and distant had lesion residue, for its total dose $50 \mathrm{~Gy}$; The normal organ tolerance dose was taken as the reference principle of RTOG (Radiation Therapy Oncology Group) for rest radiation doses. The target region dose should be increased. Meanwhile it did not exceed the limited dose of organ at risk. The fractionated dose and segmentation method was $2 \mathrm{~Gy}$ each time, once a day, 5 times every week.

\section{Efficacy and toxicity evaluation}

What's more, medical history and physical examination, blood test, chemistry profile, bone scanning, chest X-ray or chest CT, abdominal/ pelvic B ultrasound or CT, nasopharynx and neck MRI and other discomfort symptom parts were checked correspondingly. From the beginning of chemotherapy to 4 weeks after the end of chemotherapy, blood test was taken for 2-3 times every a week, chemistry profile and physical condition assessment had been tested once. Lesion was reviewed every 4 weeks. One month later, chemistry profile and lesion were tested every 2-3 months. Two years later, chemistry profile and lesion were tested every 4-6 months. If new symptoms appeared on patients, the corresponding examinations were performed at any time. The primary endpoint was overall survival (OS). The second endpoints were progression-free survival (PFS), objective response rate, relapse or progression rate in radiation field and toxicity. The objective response rate was evaluated by RECIST1.1 version. The toxicity of chemotherapy was evaluated once every cycle by NCI-CTC version 3 . The acute or chronic radiotherapy injury was evaluated by RTOG radiation toxicity criteria.

\section{Statistical analysis}

Statistical analysis was performed by using SPSS 17.0 software. PFS and OS evaluations were analyzed by Kaplan-Meier analysis. The log-rank test was compared between PFS and OS in two groups. The objective response rate, relapse or progression rate in radiation field, toxicity and other counting data were tested using Fisher's exact test in descriptive statistics analysis. The measurement data such as average age was tested by $\mathrm{T}$ test. $p<0.05$ was considered statistically significant.

\section{Follow up}

The last follow-up time was March 27th 2015. Median

Table 2. Best Response According to RECIST n (\%)

\begin{tabular}{lcccrcc}
\hline & \multicolumn{3}{c}{ after chemotherapy } & \multicolumn{3}{c}{ three months after radiotherapy } \\
& $\begin{array}{c}\text { study group } \\
(\mathrm{n}=52)\end{array}$ & $(\mathrm{n}=51)$ & value & $\begin{array}{c}\text { study group } \\
(\mathrm{n}=20)\end{array}$ & $\begin{array}{c}\text { control group } \\
(\mathrm{n}=16)\end{array}$ & $\begin{array}{c}p \\
\text { value }\end{array}$ \\
\hline $\mathrm{CR}$ & $10(19.2)$ & $2(3.9)$ & 0.03 & $17(85)$ & $8(50)$ & 0.03 \\
$\mathrm{PR}$ & $35(67.3)$ & $27(53.0)$ & 0.16 & $2(10)$ & $5(31.3)$ & 0.20 \\
$\mathrm{SD}$ & $3(5.8)$ & $17(33.3)$ & 0.00 & $3(18.7)$ & 0.08 & \\
PD & $4(7.7)$ & $5(9.8)$ & $1(5)$ & 0.00 & 1.00 & 0.30 \\
OR & $45(86.5)$ & $29(56.9)$ & 0.00 & $19(95)$ & $13(81.3)$ & 1.00 \\
DC & $48(92.3)$ & $46(90.2)$ & 0.74 & $19(95)$ & $16(100)$ & 1.00 \\
\hline
\end{tabular}

Abbreviations: CR: complete response, PR: partial response, SD: stable disease, PD: progressive disease, OR: objective response, DC: disease control 
Yong Liang et al

follow-up was 30 months. 40 patients (77\%) died of tumor progression and 12 survived in study group. Among them, 3 patients survived for more than 3 years. 44 patients $(86.3 \%)$ died of tumor progression and 7 survived in control group. Among them, 1 patient survived for about 3 years.

\section{Results}

\section{Evaluation for best response}

The number of patients in study group who completed 6 cycles was significantly higher than that of control group ( $90.4 \%$ vs $74.5 \%, p=0.04)$. Of 20 patients in study group underwent radiotherapy, whose 2-5 parts received radiotherapy, such as nasopharynx and neck, liver, lung , humerus and lumbar. There were a total of 63 parts and the average was 3.15 parts. 16 patients in control group underwent radiotherapy, whose 2-4 parts received radiotherapy in each patient and a total of 48 parts and the average was 3 parts. Local and distant lesion residual rates in control group were high after chemotherapy. So the cases and average radiotherapy doses of lesion in study group and control group respectively are: nasopharynx and neck: 20 (58Gy) vs 16 (52.5Gy) ; liver:15 (35.7Gy) vs 9 (40.9Gy); lung:6 (49Gy) vs 5 (50.4Gy); para-aortic lymph node:3 (48Gy) vs 1 (48Gy); thoracic vertebra:6 (40Gy) vs 5 (40Gy); lumbar vertebra:10 (40Gy) vs 7 (40Gy); pelvis:4 (40Gy) vs 3 (40Gy); humerus:1 (40Gy) vs 0 and femur1 (40Gy) vs 2 (40Gy).

The objective response rate was assessed by RECIST 1.1 version after chemotherapy or radiotherapy. The complete response rate and the objective response rate in study group were higher for $15.3 \%$ and $29.6 \%$ than those of control group, which had statistical significance. The primary tumor bed of complete response or residual lesions was assessed after radiotherapy for 3 months. The complete response rate was also higher than those of control group (Table 2). In addition, in-field recurrence or progression of 26 parts appeared during follow-up period in 63 radiotherapy parts in study group $(41.3 \%)$. While in-field recurrence or progression of 32 parts appeared in 48 radiation parts in control group $(66.7 \%)$. There was statistically significant in-field recurrence or progression rate between two groups, $P=0.01$.

Evaluation of PFS

The median PFS in study and control group was 20.9
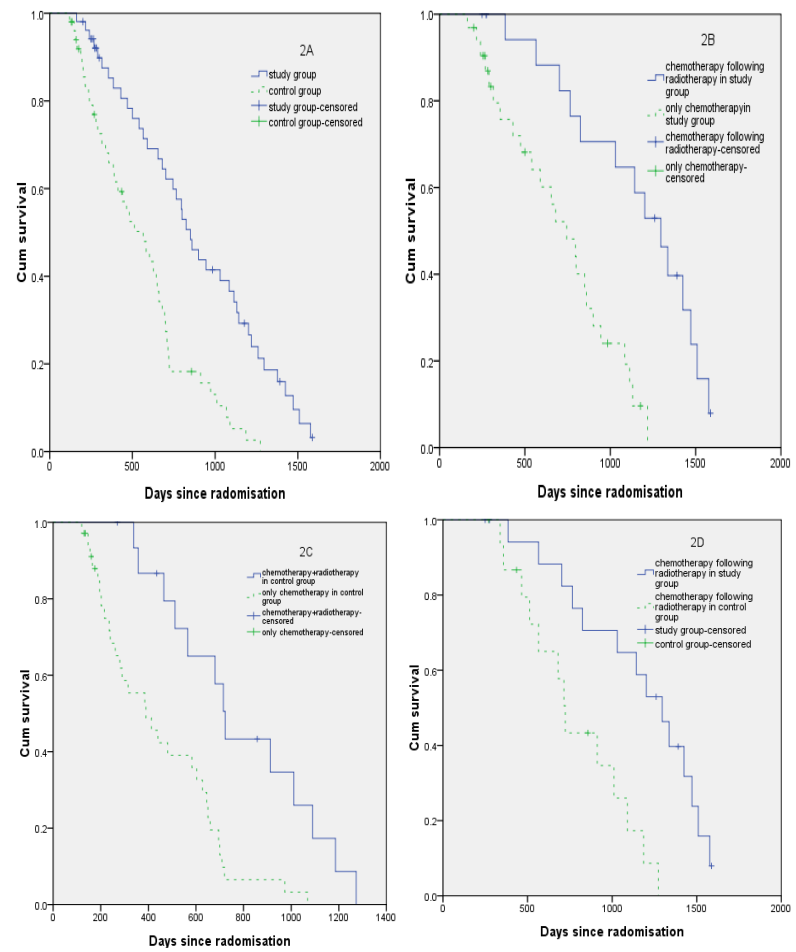

Figure 2. Kaplan-Meier Cure of Overall Survival, 2A Comparisons between study and Control Group, 2B Comparisons between Chemotherapy Following Radiotherapy and Only Chemotherapy in Study Group, 2C Comparisons between Chemotherapy Following Radiotherapy and Only Chemotherapy in Control Group, 2D comparisons between Chemotherapy Following Radiotherapy in Study and Control Group

Table 3. The Commonly Acute Treatment-Related Adverse Reactions n(\%)

\begin{tabular}{|c|c|c|c|c|c|c|}
\hline & \multicolumn{3}{|c|}{ All adverse reactions } & \multicolumn{3}{|c|}{ Grade $3-4$ adverse reactions } \\
\hline & Study group & Control group & $p$ Value & Study group & Control group & $p$ Value \\
\hline & $\mathrm{n}=52$ & $\mathrm{n}=51$ & & $\mathrm{n}=52$ & $\mathrm{n}=51$ & \\
\hline Anemia & $50(96.2)$ & $43(84.3)$ & 0.05 & $5 \quad(9.6)$ & 0.00 & 0.06 \\
\hline Granulopenia & $30(57.7)$ & $26(51.0)$ & 0.56 & $4 \quad(7.7)$ & 0.00 & 0.12 \\
\hline Thrombocytopenia & $50(96.2)$ & $21(42.2)$ & 0.00 & $7(13.5)$ & 0.00 & 0.01 \\
\hline Oral mucositis & $51(98.1)$ & $21(41.2)$ & 0.00 & $27(51.9)$ & 12.00 & 0.00 \\
\hline Vomit & $39(75.0)$ & $35(68.6)$ & 0.52 & $3(5.8)$ & $1(2.0)$ & 0.62 \\
\hline Nausea & $51(98.1)$ & $47(92.2)$ & 0.21 & $2(3.8)$ & 0.00 & 0.50 \\
\hline Loss of appetite & $50(96.2)$ & $35(68.6)$ & 0.00 & $6(11.5)$ & 0.00 & 0.03 \\
\hline Fatigue & $38(73.1)$ & $16(31.4)$ & 0.00 & $11(12.9)$ & $2(2.4)$ & 0.02 \\
\hline Diarrhea & $5 \quad(9.6)$ & $3(5.9)$ & 0.72 & 0.00 & 0.00 & \\
\hline Increased aminopherase & $18(34.6)$ & $15(29.4)$ & 0.67 & $2(3.8)$ & 0.00 & 0.50 \\
\hline Increased bilirubin & $6(11.5)$ & $2(3.9)$ & 0.27 & 1 (1.9) & 0.00 & 1.00 \\
\hline Increased serum creatinine & $5 \quad(9.6)$ & $3(5.9)$ & 0.72 & 0.00 & 0.00 & \\
\hline Neurotoxicity & $5 \quad(9.6)$ & $3(5.9)$ & 0.72 & 0.00 & 0.00 & \\
\hline Ototoxicity & $3 \quad(5.8)$ & $1 \quad(2.0)$ & 0.62 & 0.00 & 0.00 & \\
\hline Skin damage & $20(38.5)$ & $9(17.6)$ & 0.03 & $4 \quad(7.7)$ & $2(3.9)$ & 0.68 \\
\hline Total cases & $52(100)$ & $45(94.0)$ & 0.01 & $28(53.8)$ & $5(9.8)$ & 0.00 \\
\hline
\end{tabular}


months [95\%CI 17.5-24.1] vs 12.7 months [95\%CI 11.214.1], which was significantly improved for 8.2 months in study group ( $p=0.00$ Figure1A). Compared with PFS in selective radiotherapy after chemotherapy, the median PFS in study and control group was 34.9 months [95\% CI 29.1-40.6] and 16.2 months [95\%CI 12.2-20.1]. The former median PFS was significantly prolonged for 18.7 months $(p=0.00$ Figure 1B)

\section{Evaluation for $O S$}

The median OS, the survival rates in 1,2 and 3 years in study and control group were 28.3 months [95\% CI 23.932.8 ] vs 18.8 months [95\% CI13.0-24.6], $85.2 \%$ vs $65.9 \%$, $62.2 \%$ vs $18.3 \%$ and $36.6 \%$ vs $5.2 \%$. The improvement in study group were statistically significant $(p=0.00,0.04$, $0.00,0.00$ respectively (Figure $2 \mathrm{~A}$ ).

Subgroup analysis showed that the median OS, the survival rates in 1, 2 and 3 years compared

chemotherapy following radiotherapy with only chemotherapy in study group were 43.2 months [95\% CI 35.0-51.3] vs 24.8 months [95\%CI17.3-32.3], $94.1 \%$ vs $75.7 \%, 82.4 \%$ vs $52.1 \%, 64.7 \%$ vs $19.2 \%$ ( $p=0.00,0.53$, $0.05,0.00$, respectively, Figure $2 \mathrm{~B})$. The median OS, the survival rates in 1, 2 and 3 years compared chemotherapy following radiotherapy with only chemotherapy in control group were 24.1 months [95\% CI 21.5-26.7] vs 13.0months [95\%CI 7.4-18.6], $86.7 \%$ vs $55.4 \%, 43.3 \%$ vs $6.5 \%, 17.3 \%$ vs $0 \%$ ( $p=0.00,0.03,0.00,0.03$, respectively, Figure2C). The chemotherapy following radiotherapy in study group was compared with that of control group, the median OS , the survival rates in 1,2 and 3 years were 43.2 months [95\%CI 35.0-51.3] vs 24.1 months [95\%CI 21.5-26.7], $94.1 \%$ vs $86.7 \%, 82.4 \%$ vs $43.3 \%, 64.7 \%$ vs $17.3 \%$ ( $p=0.00,0.57,0.04,0.01$ respectively, Figure 2D).

\section{Acute and late toxicities}

The treatment related death that did not occur on all patients in the course of treatment. The total and grade 3-4 acute adverse reaction rates were $100 \%$ and $53.8 \%$ in study group, which was $6 \%$ and $44 \%$ higher than those of control group. The difference was statistically significant. The total adverse reactions including thrombocytopenia, oral mucositis, loss of appetite, fatigue, skin damage, 3-4 level adverse reactions including thrombocytopenia, oral mucositis, loss of appetite, fatigue were increased (Table 3 ). But the difference of chronic toxicity of radiotherapy had no statistically significant between study and control groups such as grade 1-2 xerostomia $40 \%$ vs $44 \%$, grade $1-2$ radiation related pneumonia $50 \%$ v $40 \%$ (all $p>0.05$ ).

\section{Discussion}

To the best of our knowledge, this is the first clinical study on patients with distant metastases of NPC for dose-dense chemotherapy of cisplatin plus fluorouracil following selection radiotherapy to show an improvement in efficacy outcomes. Dose-dense chemotherapy refers to shorten the inter-treatment interval to minimize the regrowth of tumor cells, thus allowing for more effective cell killing (Hudis et al., 2015). The higher complete response rate was obtained in study group by using dose-dense cisplatin combined with fluorouracil chemotherapy, so more complete response patients received radiotherapy that relapse or progression rate in radiation field, median PFS, median OS, survival rate in 2 and 3 years of whole or patients undergoing radiotherapy in study group were significantly better than those of control group. The possible mechanisms to improve the effect included: A. Dose-dense chemotherapy reduced the production of secondary drug resistant tumor cells, which is the main reason for the failure of chemotherapy. During the period of cell proliferation drug resistant cells can be generated, which are positively related with doubling times, therefore shortening chemotherapy interval can reduce the production of drug resistance cells, which can make drug eliminate cancer cells more thoroughly. B. To reduce tumor proliferation time. Tumor cell re-proliferation appears during chemotherapy interval. The longer the time is, the more the proliferation is. The chemotherapy cycle was shortened from 28 days to 14 days. In case of the doubling time of stem cell was 24 hours. Dosedense chemotherapy could reduce the production of a tumor stem cell from $2^{28}$ to $2^{14}$ cells (reduce16384times). Although this pure theory value, which does not take the loss of tumor cells and stem cells which has turned to G0 or non proliferation cells into account, was higher than the actual situation. The complete response rate of dose-dense chemotherapy $19.2 \%$ was significantly higher than 3.9\% in control group whose chemotherapy cycles were 28 days. This could explain the effect of reducing tumor proliferation time on size of tumor. C. Dose-dense chemotherapy residual tumor proliferation time was reduced. The tumor volume was smaller, but the growth proportion was increased. The cell cycle specific cytotoxic drugs such as cisplatin play a great role in the treatment. D. The tumor volume was decreased, which increased patients' confidence and compliance. The chemotherapy plan was easy to be completed. $90.4 \%$ of dose-dense chemotherapy patients completed 6 cycles of chemotherapy, but only $74.5 \%$ of patients completed chemotherapy in control group. Fandi (2000) advocates 6 cycles of chemotherapy for NPC patients with distant metastases because less cycles would affect treatment effect. E. Dose-dense chemotherapy could kill potential dispersed subclinical lesions more effectively. Therefore, the median OS in study group and patients who received dose-dense chemotherapy following radiotherapy in this group was prolonged for 9.5 and 19.1 months than that of control group. F. Dose-dense chemotherapy obtained higher complete response rate and objective response rate. The more cytoreduction cells were, the less residual tumor cells were. At the same time chemotherapy had little effect on blood vessel supplying tumor so hypoxia tumor cells obtained more oxygen led them more sensitive to radiotherapy that significantly reduced the recurrent or progressive rate in radiation field, and increased the overall survival rate in 2 and 3 years.

First of all, a number of clinical literatures in recent years show that some patients with non dissemination distant metastasis achieved long-term survival after radiotherapy. (Lutz et al., 2014; Nagamata et al., 2014; 
Yong Liang et al

Guenne et al., 2014; Hingorani et al., 2015). Secondly, neoadjuvant chemotherapy based on cisplatin could improve local control and survival rate in radiotherapy of locally advanced NPC (Xu et al., 2012; Du et al., 2013; Kong et al., 2014). The radiotherapy of nasopharynx, neck and/ or bone metastases to treat the NPC patients with distant metastases could prolong complete response time and increase the survival rate after systematic chemotherapy (Chen et al., 2013; Agnese et al., 2014). Thirdly, NPC was sensitive to chemotherapy. The objective response rate of standard first-line schedule cisplatin combination with fluorouracil in the treatment of distant metastasis of NPC was $66-78 \%$ (Bensouda et al., 2011). The primary/ secondary drug resistance led to unsatisfactory total survival time. While the NPC cells were more sensitive to radiotherapy than chemotherapy, and radiotherapy remained valid on chemotherapy resistant patients (Pandey et al., 2013); Thus a corollary could be drawn: if subclinical lesions with diffuse metastasis was eliminated on distant metastasis of NPC patients by chemotherapy, then the radiotherapy effect on tumor bed would be further increased OS and even some patients obtained long-term survival. To eliminate the subclinical lesions with diffuse metastasis, intensive chemotherapy was the most commonly used methods. The intensive chemotherapy included dose-intensity and shortened chemotherapy dose-density (Peto et al., 2012). Dose-intensity chemotherapy in head and neck carcinoma failed to apply because of a randomized trial comparing $60 \mathrm{mg} / \mathrm{m}^{2}$ vs $120 \mathrm{mg} / \mathrm{m}^{2}$ of cisplatin failed to demonstrate a significant improvement in response and survival (Veronesi et al., 1985) and another trial that evaluated very high doses of cisplatin up to $200 \mathrm{mg} / \mathrm{m}^{2}$ failed to complicat by severe toxicities. (Havlin et al., 1989). Otherwise dose-density chemotherapy has made a significant contribution to the adjuvant treatment of breast cancer (Hudis et al., 2015). NPC was also sensitive to chemotherapy, whether dose-dense chemotherapy could bring the same clinical benefit as patients with breast cancer? Our study showed that the CR rate was $19.2 \%$ after dose-dense chemotherapy, which was higher than $15 \%$ reported by Jamshed (2014), and was significantly higher than that (3.9\%) of traditional chemotherapy. The rate of CR (3.9\%) and PR (53.0\%) in control group was similar with $4.3 \%$ and $51.7 \%$ reported by Zeng (2014). The CR rate $85 \%$ of patients following radiotherapy was higher than $50.0 \%$ in control group and $64.3 \%$ reported in the literature (Kang et al. 2013) after dose-dense chemotherapy. And the median 0S, survival rate in 2 and 3 year were increased for 19.1 months, $39.1 \%$ and $47.4 \%$ than control group. These results suggested that dosedense chemotherapy following selection radiotherapy was beneficial on the patients with distal metastasis of NPC.

The acute adverse reaction was increased obviously after dose-dense chemotherapy. The grade 3-4 adverse reaction reached as high as $53.8 \%$, especially thrombocytopenia, oral mucositis, loss of appetite and fatigue, but patients could tolerate it. The other adverse reactions were non significantly difference between study and control group such as the transaminase was increased by $34.6 \%$ and bilirubin was increased by $11.5 \%$ after liver radiotherapy, which was similar with Yin ( 2014).

The risk of neutropenia would increase significantly on patients after dose-dense chemotherapy. Serious neutropenia will cause chemotherapy delay, chemotherapy reduction or chemotherapy related infection was also increased (Aapro et al., 2011). But the severe neutropenia did not appear in our research. This was because of preventive use of recombinant human granulocyte colony stimulating factor (Crawford et al; 2010) from day6 to day 10. Although the acute adverse reaction was increased obviously in study group, late radiotherapy toxicity did not increase. For example, the grade 1-2 xerostomia was $40 \%$, which was similar with Li (2014).

Limitations: Firstly, chemotherapy was phase III clinical trial, but it was a single-center study and the number of samples was small. Secondly, the patients were randomly divided by using digital random table, but they were not stratified by metastatic site, primary treatment and history of chemotherapy drugs, dose of radiotherapy and performance status. This method would led to bias. Thirdly, the number of patients undergoing radiotherapy was less, radiotherapy position was not completely consistent with dose; Fourthly, radiotherapy was compared with only chemotherapy after intragroup chemotherapy, the former OS was significantly prolonged, but radiotherapy patients were those with good response to chemotherapy and the lesions were not dissemination.

\section{Acknowledgements}

This research was supported by Science and Technology Bureau of Guigang City, China (0807004).

\section{References}

Aapro MS, Bohlius J, Cameron DA, et al (2011). 2010 update of EORTC guidelines for the use of granulocytecolony stimulating factor to reduce the incidence of chemotherapy induced febrile neutropenia in adult patients with lymphoproliferative disorders and solid tumours. Eur J Cancer, 47, 8-32.

Agnese D, Belgioia L, Bacigalupo A, et al (2014). Prolonged complete remission after induction chemotherapy followed by chemoradiation with tomotherapyin metastatic nasopharyngeal cancer. Anticancer Res, 34, 5075-8.

Abbasi AN, Hafiz A, Ali N, et al (2013). Plan dose evaluation of 3D-CRT of nasopharyngeal cancer in Pakistan. Asian Pac J Cancer Prev, 14, 5989-93.

Aoki H, Ogura R, Tsukamoto Y, et al (2013). Advantages of dosedense methotrexate protocol for primary central nervous system lymphoma: comparison of two different protocols at a single institution. Neurol Med Chir, 53, 797-804.

Bensouda Y, Kaikani W, Ahbeddou N, et al (2011). Treatment for metastatic NPC. european annals of otorhinolaryngology, Head and Neck Diseases, 128, 79-85.

Chen MY, Jiang R, Guo L, et al (2013). Locoregional radiotherapy in patients with distant metastases of NPC at diagnosis. Chin J Cancer, 32, 604-13.

Crawford J, Caserta C, Roila F, et al (2010). Hematopoietic growth factors: ESMO clinical practice guidelines for the applications. Ann Oncol, 21, 248-51.

Du C, Ying H, Zhou J, et al (2013). Experience with combination of docetaxel, Cisplatin plu-s 5-fluorouracil chemotherapy, and intensity-modulatedradiotherapy for locoregionally 

advanced NPC. Int J Clin Oncol, 18, 464-71.

Guenne C, Fayette J, Cosmidis A, et al (2014). Curative treatment can be an option for patients with metastatic squamous cell cancer of the head and neck. Drug Des Devel Ther, 8 , 2549-53.

Fandi A, Bachouchi M, Azli N, et al (2000). Long-term diseasefree survivors in meta-static undifferentiated carcinoma of nasopharyngeal type. J ClinOncol, 18, 1324-30.

Havlin KA1, Kuhn JG, Myers JW, et al (1989). High-dose cisplatin for locally advanced or met-astatic head and neck cancer. A phase II pilot study. Cancer, 63, 423-7.

Hingorani M, Dixit S, Johnson M, et al (2015). Palliative radiotherapy in the presence of well-controlled metastatic disease after Initial chemotherapy may prolong survival in patients with metastatic esophageal and gastric cancer. Cancer Res Treat.

Hudis C, Dang C (2015). The development of dose-dense adjuvant chemotherapy. Breast J, 21, 42-51.

Jamshed A, Hussain R, Iqbal H. (2014). Gemcitabine and cisplatin followed by chemo-radiati-on in advanced NPC. Asian Pac J Cancer Prev, 15, 899-904.

Jemal A, Bray F, Center MM, et al (2011). Global cancer statistics. CA Cancer J Clin, 61, 69-90.

Kang M, Liu WQ, Qin YT, et al (2013). Long-term efficacy of microwave hyperthermia combined with chemoradiotherapy in treatment of NPC with cervical lymph node metastases. Asian Pac J Cancer Prev, 14, 7395-400

Kertmen N, Aksoy S, Cengiz M, et al (2015). Comparison of three different induction regimens for nasopharyngeal cancer. Asian Pac J Cancer Prev, 16, 59-63.

Kong FF, Ying H, Du CR, et al (2014). Effectiveness and toxicities of intensity-modulated radiation therapy for patients with T4 nasopharyngealcarcinoma. PLoS One, $\mathbf{9}$, 91362.

Kua VF, Ismail F, Chee Ee Phua V, et al (2013). Carboplatin/5fluorouracil as an alternat-ive to cisplatin/5- fluorouracil for metastatic and recurrent head and necksquamous cellcarcinoma and NPC. Asian Pac J Cancer Prev, 14, 1121-6.

Kumar A, Hoskins PJ, Tinker AV (2015). Dose-dense paclitaxel in advanced ovarian cancer. Clin Oncol ( $R$ Coll Radiol), 27, 40-7.

Lee AW, Ng WT, Chan YH, et al (2012) . The battle against nasopharyngeal cancer. Radiother Oncol, 104, 272-8.

Li MY, Liu JQ, Chen DP, et al (2014). Glycididazole sodium combined with radiochemothe- rapy for locally advanced NPC. Asian Pac J Cancer Prev, 15, 2641-6.

Lutz ST, Jones J, Chow E (2014). Role of radiation therapy in palliative care of the patient with cancer. J Clin Oncol, 32, 2913-9.

Nagamata M, Okuma Y, Yamada Y, et al (2014). Crucial role of treatment with palliative intent for a patient with advanced thymic carcinoma. OncolLett, 8, 513-6.

$\mathrm{Ng}$ WT, Lee MC, Hung WM, et al (2011). Clinical outcomes and patterns of failure after intensity-modulated radiotherapy for NPC. Int J Radiat Oncol Biol Phys, 79, 420-8.

Pandey KC, Revannasiddaiah S, Pant NK, et al (2013). Palliative radiotherapy in locally advanced head and neck cancer after failure of induction chemotherapy:comparison of two fractionation schemes. Indian J Palliat Care, 19, 139-45.

Peto R, Davies C, Godwin J, et al (2012). Comparisons between different poly Chemotherapy regimens forearly breast cancer: meta-analyses of long-term outcome among 100, 000 women in 123 randomised trials. Lancet, 379, 432-44.

Veronesi A, Zagonel V, Tirelli U, et al (1985). High-dose versus low-dose cisplatin in advanced head and neck squamous carcinoma: a randomized study. J Clin Oncol, 3, 1105-8.
Wang TL, Tan YO (1991). Cisplatin and 5-fluorouracil continuous Infusion for metastaticNPC. Ann Acad Med Singapore, 20, 601-3.

Xiao WW, Huang SM, Han F, et al (2011). Local control, survival, and late toxicities of locally advanced NPC treated by simultaneous modulated accelerated radiotherapy combined with cisplatin concurrent chemotherapy: longterm results of a phase 2 study. Cancer, 117, 1874-83.

Xu T, Hu C, Zhu G, et al (2012). Preliminary results of a phase III randomized studycomparing chemotherapy neoadjuvantly or concurrently withradiotherapy for locoregi-onally advanced NPC. Med Oncol, 29, 272-8.

Yin H, Lu K, Qiao WB, et al (2014). Whole-liver radiotherapy concurrent with chemotherapy as a palliative treatment for colorectal patients withmassive and multiple liver metastases: a retrospective study. Asian Pac J Cancer Prev, 15, 1597-602.

Zeng L, Tian YM, Huang Y, et al (2014). Retrospective analysis of 234 NPC patients with distant metastasis at initial diagnosis:therapeutic approaches and prognostic factors. PLoS One, 9, 108070. 